\title{
Dihydrolipoic acid protects against lipopolysaccharide-induced behavioral deficits and neuroinflammation via regulation of $\mathrm{Nrf2/HO}-1 / \mathrm{NLRP3}$ signaling in rat
}

Hetao Bian, Gaohua Wang*, Junjie Huang, Liang Liang, Yage Zheng, Yanyan Wei, Hui Wang, Ling Xiao and Huiling Wang

\begin{abstract}
Background: Recently, depression has been identified as a prevalent and severe mental disorder. However, the mechanisms underlying the depression risk remain elusive. The neuroinflammation and NLRP3 inflammasome activation are known to be involved in the pathology of depression. Dihydrolipoic acid (DHLA) has been reported as a strong antioxidant and exhibits anti-inflammatory properties in various diseases, albeit the direct relevance between DHLA and depression is yet unknown. The present study aimed to investigate the preventive effect and potential mechanism of DHLA in the lipopolysaccharide (LPS)-induced sickness behavior in rats.

Methods: Adult male Sprague-Dawley rats were utilized. LPS and DHLA were injected intraperitoneally every 2 days and daily, respectively. Fluoxetine (Flu) was injected intraperitoneally daily. PD98059, an inhibitor of ERK, was injected intraperitoneally $1 \mathrm{~h}$ before DHLA injection daily. Small interfering ribonucleic acid (siRNA) for nuclear factor erythroid 2-like (Nrf2) was injected into the bilateral hippocampus 14 days before the DHLA injection. Depression-like behavior tests were performed. Western blot and immunofluorescence staining detected the ERK Nrf2/HO-1/ROS/NLRP3 pathway-related proteins.

Results: The DHLA and fluoxetine treatment exerted preventive effects in LPS-induced sickness behavior rats. The DHLA treatment increased the expression of ERK, Nrf2, and HO-1 but decreased the ROS generation levels and reduced the expression of NLRP3, caspase-1, and IL-1 $\beta$ in LPS-induced sickness behavior rats. PD98059 abolished the effects of DHLA on preventive effect as well as the levels of Nrf2 and HO-1 proteins. Similarly, Nrf2 siRNA reversed the preventive effect of DHLA administration via the decreased expression of HO-1.
\end{abstract}

Conclusions: These findings suggested that DHLA exerted a preventive effect via ERK/Nrf2/HO-1/ROS/NLRP3 pathway in LPS-induced sickness behavior rats. Thus, DHLA may serve as a potential therapeutic strategy for depression.

Keywords: Dihydrolipoic acid, Neuroinflammation, NLRP3, Lipopolysaccharide, Depression

\footnotetext{
*Correspondence: wgh6402@163.com

Department of Psychiatry, Renmin Hospital of Wuhan University, Jiefang

Road 238\#, Wuhan 430060, Hubei, PR China
}

(c) The Author(s). 2020 Open Access This article is licensed under a Creative Commons Attribution 4.0 International License, which permits use, sharing, adaptation, distribution and reproduction in any medium or format, as long as you give appropriate credit to the original author(s) and the source, provide a link to the Creative Commons licence, and indicate if changes were made. The images or other third party material in this article are included in the article's Creative Commons licence, unless indicated otherwise in a credit line to the material. If material is not included in the article's Creative Commons licence and your intended use is not permitted by statutory regulation or exceeds the permitted use, you will need to obtain permission directly from the copyright holder. To view a copy of this licence, visit http://creativecommons.org/licenses/by/4.0/ The Creative Commons Public Domain Dedication waiver (http://creativecommons.org/publicdomain/zero/1.0/) applies to the data made available in this article, unless otherwise stated in a credit line to the data. 


\section{Background}

Depression is the most common psychiatric mood disorder globally. Currently, the limited therapies available for the treatment of this condition mainly target the monoamine levels. However, this approach is not efficient for many patients [1]. The mechanisms underlying depression are complicated and largely unknown. Therefore, the knowledge of this mechanism of depression would aid the development of effective treatment.

Accumulating evidence revealed a close link between inflammation and major depression disorder $[2,3]$. The nod-like receptor pyrin-containing pyrin domain 3 (NLRP3) inflammasome is one of the most widely studied inflammasomes [4]. After NLRP3 inflammasome is assembled and activated, it activates caspase- 1 by proteolytic cleavage, which in turn, converts pro-IL- $1 \beta$ into bioactive IL-1 $\beta$, leading to inflammatory responses in bodies $[5,6]$. Reactive oxygen species (ROS) is a normal metabolic product of redox reactions. The excessive level of ROS would damage the integrity of cells and result in dysfunction of the tissues via peroxidation of lipids, proteins, mitochondria, and DNA of cells $[7,8]$. Despite that the specific regulatory mechanism of NLRP3 inflammasome activation is unclear, ROS has been frequently reported to be correlated with NLRP3 inflammasome activation $[9,10]$. Also, it regulates the expression and/ or activation of NLRP3 inflammasome in several diseases, including intestinal inflammation and cardiovascular disease.

Microglia, as resident immune cells which fulfill different tasks, are mainly involved in the inflammatory response and in maintaining homeostasis within the central nervous system (CNS). Microglial activation is the principal component of neuroinflammation in the CNS, the function of microglial cells in the pathophysiology of depression has attracted more and more attention [11].

Nuclear factor erythroid 2-like (Nrf2) is a primary element of transcription and has emerged as a potential therapeutic target for inflammatory disorders [12]. Heme oxygenase-1 (HO-1) is an enzyme that exerts antiinflammatory and antioxidant stress effects [13]. Nrf2 is a critical modulator of the expression of HO-1 [14, 15]. Recent studies have focused on the Nrf2/HO-1 approach with respect to anti-inflammation $[16,17]$. These findings indicated that the $\mathrm{Nrf} 2 / \mathrm{HO}-1$ signaling pathway plays a critical role in anti-inflammatory activities. Other studies have shown that the activated Nrf2/HO-1 signaling pathway may counteract the intracellular production of ROS [18].

Dihydrolipoic acid (DHLA) is a reduced form of $\alpha$ lipoic acid (LA) that can decrease oxidative stress and act as a strong antioxidant. DHLA also possesses antiinflammatory properties [19]. Hitherto, the preventive effect of DHLA has not been explored. Therefore, in the present study, we sought to investigate the preventive effect of DHLA in the LPS-induced sickness behavior animal model and whether ERK/Nrf2/HO-1/ROS/NLRP3 pathway is involved in the preventive effect of DHLA.

\section{Methods \\ Animals}

Adult male Sprague-Dawley (SD) rats (weight, 200-220 g) were purchased from Hunan SJA Laboratory Animal Co., Ltd (Hunan, China) and housed in a $12 \mathrm{~h}$ dark and light cycle at room temperature $\left(18-22^{\circ} \mathrm{C}\right)$ with free access to water and food. All procedures involving animals were approved and carried out according to the guidelines of the Institutional Animals Care Committee of Renmin Hospital of Wuhan University.

\section{Experimental design}

This study consisted of four experiments as shown in Fig. 1.

Experiment 1: Rats were randomly divided into six groups ( $n=6 /$ group): Control, Lipopolysaccharide (LPS) + vehicle, LPS + DHLA (15 mg/kg, $30 \mathrm{mg} / \mathrm{kg}, 60 \mathrm{mg} / \mathrm{kg})$, LPS+ fluoxetine (Flu) group. The evaluation of the body weight, open field test (OFT), and forced swim test (FST) was used to assess the anti-depression effects of DHLA. Based on the body and behavioral tests, $30 \mathrm{mg} /$ $\mathrm{kg}$ DHLA-treated group was selected for the subsequent experiments.

Experiment 2: Rats were randomly divided into four groups ( $n=9$ /group): Control, LPS, LPS + vehicle, and LPS + DHLA (30 mg/kg). The expression of ERK, Nrf2, and HO-1 was detected by Western blot, while that of ROS was tested by flow cytometry ( $n=6$ /group). Immunofluorescence staining assessed the expression of HO-1 ( $n=3$ /group). The test of body weight, OFT, and FST was used to assess the anti-depression effects of DHLA.

Experiment 3: Rats were randomly divided into six groups ( $n=6$ /group): Control, LPS, LPS+ vehicle, LPS + DHLA (30 mg/kg), LPS + DHLA (30 mg/kg) + DMSO, LPS + DHLA $(30 \mathrm{mg} / \mathrm{kg})+$ PD98059. The expression of ERK, Nrf2, HO-1, NLRP3, caspase-1, and IL-1 $\beta$ was detected by Western blot. The test of body weight, OFT, and FST was used to assess the anti-depression effects of DHLA.

Experiment 4: Rats were randomly divided into six groups ( $n=6 /$ group): Control, LPS, LPS+ vehicle, LPS + DHLA (30 mg/kg), LPS + DHLA (30 mg/kg) + AAV control-siRNA, LPS + DHLA (30 mg/kg) + AAV-Nrf2siRNA. The expression of ERK, Nrf2, HO-1, NLRP3, caspase-1, and IL-1 $\beta$ was detected by Western blot. The test of body weight, OFT, and FST was used to assess the anti-depression effects of DHLA. 


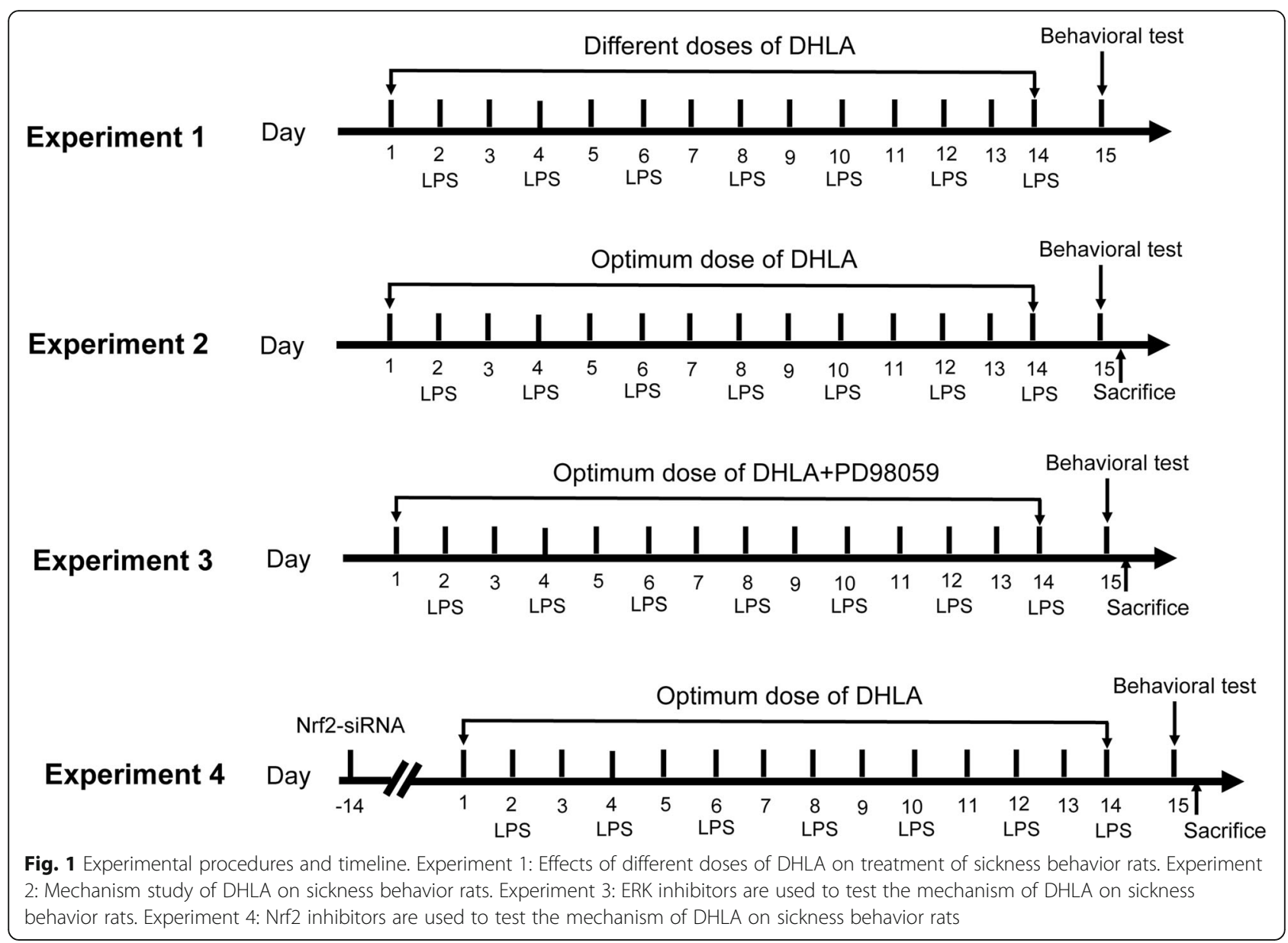

\section{Drug treatment}

LPS (Escherichia coli 055:B5, Sigma) was solubilized in dimethyl sulfoxide (DMSO) and phosphate-buffered saline (PBS) and administered intraperitoneally (i.p.) at a dosage of $500 \mu \mathrm{g} / \mathrm{kg}$ every 2 days as described previously [20]. DHLA (Sigma) was diluted in DMSO and PBS and administered intraperitoneally (i.p.) daily to a total of 14 injections. The ERK antagonist PD98059 (MedChemExpress, $0.3 \mathrm{mg} / \mathrm{kg}$ ) was diluted in DMSO and PBS and administered intraperitoneally (i.p.) daily [21]. Flu (Aladdin Reagent Shanghai) was solubilized in sterile distilled water and administered intraperitoneally (i.p.) at a dosage of $10 \mathrm{mg} / \mathrm{kg} /$ day [22].

\section{Hippocampal administration}

Adeno-associated virus-mediated small interfering RNA against Nrf2 (AAV-Nrf2-siRNA) or control vector (AAV-Control-siRNA) with an enhanced green fluorescent protein (eGFP) was purchased from Genechem Co., Ltd. (Shanghai, China). The siRNA sequence for Nrf2 is 5'-GTCTTCAGCATGTTACGT GATGAGGATGG-3' [23]. Hippocampal AAV virus administration was performed as described previously
[24]. Briefly, rats were anesthetized with $10 \%$ chloral hydrate $(0.35 \mathrm{~mL} / 100 \mathrm{~g}$, i.p. $)$ and placed in a stereotaxic apparatus. Rats were infused bilaterally with $1 \mu \mathrm{L}$ of purified and concentrated AAV virus $(1.08 \times$ $10^{13} \mathrm{v} . \mathrm{g} / \mathrm{mL}$ ) into the hippocampus region (coordinates from the bregma: $-3.5 \mathrm{~mm}$ posterior, $\pm 2.3 \mathrm{~mm}$ lateral, $-3.0 \mathrm{~mm}$ ventral) using an electric microinjection pump (Stoelting, USA). The needle was kept in place for $5 \mathrm{~min}$ after infusion and then removed slowly. Subsequently, the incision was closed with interrupted silk sutures, and the animal was placed in a heated cage $\left(35^{\circ} \mathrm{C}\right)$ and monitored carefully.

FST

FST was performed as described previously. The rats were singly placed in glass cylinders $(40-\mathrm{cm}$ height and 28 - $\mathrm{cm}$ diameter) filled with $30 \mathrm{~cm}$ of water $\left(25 \pm 1{ }^{\circ} \mathrm{C}\right)$ for $15 \mathrm{~min}$ for training, and after $24 \mathrm{~h}$, the animals were placed again in the cylinders for $6 \mathrm{~min}$. The immobile time was recorded during the final $4 \mathrm{~min}$. The immobility was defined as floating with only minimal movements to maintain their head above water. 
Oft

OFT was performed to measure spontaneous activity, as described previously [25]. Briefly, each test rat was placed in the apparatus consisting of a black square 100 $\mathrm{cm} \times 100 \mathrm{~cm}$. The evaluations of every rat were recorded for $5 \mathrm{~min}$, and the rat movements were recorded by a video tracking system (Ethovision XT 11.5). The frequencies of rearing, total distance, and total speed were analyzed using the video tracking system. The apparatus was cleaned with $70 \%$ alcohol after each test.

\section{Flow cytometry}

Intracellular oxidative stress was determined by flow cytometry using a ROS assay kit (Jiancheng Biotechnology, Nanjing, China) according to the manufacturer's protocol [26]. 2,7-Dichlorofluorescein-diacetate (DCFH-DA) was utilized as a sensitive nonfluorescent precursor dye. It permeates the cells and is hydrolyzed by intracellular esterase to the nonfluorescent DCFH, which is rapidly oxidized to the highly fluorescent 2,7-dichlorofluorescein (DCF) in the presence of ROS. The fluorescence intensity of DCF was proportional to the level of intracellular ROS as measured by flow cytometry.

\section{Western blot analysis}

Western blot analysis was performed as described previously [27]. One day after the behavioral tests, the rats were anesthetized with $10 \%$ chloral hydrate $(3.5 \mathrm{~mL} / 100$ g), and the brains were collected. Total protein was prepared from the hippocampus, and the BCA assay was used to analyze the concentration of proteins (BCA Protein Assay, Thermo, 23228). The protein sample was resolved on SDS-PAGE and then transferred to a PVDF membrane. The membranes were probed overnight with the following primary antibodies at $4{ }^{\circ} \mathrm{C}$ : Nrf2 (Abcam, ab137550; 1:1000), HO-1 (Abcam, ab13243; 1:1000), ERK (Abcam, ab17942; 1:1000), p-ERK (Abcam, ab50011; 1:1000), NLRP3 (Abcam, ab214185; 1:1000), caspase-1 (Abcam, ab179515; 1:1000), and IL-1 $\beta$ (Abcam, ab9722; 1:1000). Subsequently, the membranes were washed with TBST ( 3 times for 5 min each) and incubated with goat anti-rabbit IgG (1:2000; Abcam, ab205718) and goat anti-mouse IgG (1:2000; Abcam, ab205719), as appropriate, for $1 \mathrm{~h}$ at room temperature. The immunoreactive bands were developed by chemiluminescence using Chemi Doc XRS System (Bio-rad, USA), and the intensities were normalized to that of GAPDH (1:10000; Servicebio, GB11002) used as an internal standard and quantified using ImageJ software.

\section{Immunofluorescence}

Immunofluorescence staining was conducted as described previously [28]. A series of $30 \mu \mathrm{m}$ slices were blocked in $2 \%$ BSA and incubated overnight with the primary antibody at $4{ }^{\circ} \mathrm{C}$. Primary antibodies: anti-HO-1 (Abcam, ab13243; 1:1000) and anti-IBA-1(Wako, 01919741; 1:1000) were used as primary antibodies. The secondary antibody was probed on the slide for $1 \mathrm{~h}$ in the dark. Subsequently, the sections were washed three times with PBS before the images were acquired using a Nikon upright fluorescence microscope.

\section{Statistical analysis}

Prism software (GraphPad Prism 8.0, CA, USA) was used for all the analyses. The data are presented as mean \pm standard error of the mean (SEM). The normality of the distribution was assessed by the Shapiro-Wilk test. Statistical comparisons were made using one-way analysis of variance (ANOVA), followed by Tukey's post hoc test. Statistical significance was indicated by $p$ value $<0.05$.

\section{Results \\ DHLA treatment reversed the LPS-induced sickness behavior}

Bodyweight gain and behavioral tests, including OFT and FST, were performed to investigate the effects of DHLA on LPS-induced sickness behavior in rats.

As shown in Fig. 2a, rats exposed to LPS showed less body weight gain as compared to the control group ( $F(5$, $30)=19.83, p<0.0001)$. However, treatment with DHLA $(30 \mathrm{mg} / \mathrm{kg}, p<0.001 ; 60 \mathrm{mg} / \mathrm{kg}, p<0.01)$ and Flu $(p<0.0001)$ improved the body weight gain as compared to the LPS group.

The FST is mainly used to measure the depressionlike behavior. As shown in Fig. 2b, rats exposed to LPS showed more immobility time in FST as compared to the control group $(F(5,30)=17.49, p<0.0001)$, whereas compared to the LPS group, DHLA $(30 \mathrm{mg} / \mathrm{kg}, p<$ $0.001 ; 60 \mathrm{mg} / \mathrm{kg}, p<0.01)$ and Flu $(p<0.0001)$ treatment markedly decreased the immobility time in FST.

The performance of rats in OFT is shown in Fig. 2c-e. The total distance, total velocity, and rearing frequencies were significantly decreased in the LPS group as compared to the control group (total distance $(F(5,30)=$ $19.93, p<0.0001)$; velocity $(F(5,30)=8.132, p<0.0001)$; rearing frequencies $(F(5,30)=11.66, p<0.0001)$. Compared to the LPS group, the total distance in the DHLA $(30 \mathrm{mg} / \mathrm{kg}, p<0.001 ; 60 \mathrm{mg} / \mathrm{kg}, p<0.01)$ and Flu $(p<$ $0.0001)$ groups was significantly increased, the total velocity in the DHLA $(30 \mathrm{mg} / \mathrm{kg}, p<0.01 ; 60 \mathrm{mg} / \mathrm{kg}, p<$ $0.05)$ and Flu $(p<0.01)$ groups were significantly increased, and the rearing frequencies in the DHLA (30 $\mathrm{mg} / \mathrm{kg}, p<0.01)$ and Flu $(p<0.01)$ groups were significantly increased.

Since DHLA $(30 \mathrm{mg} / \mathrm{kg})$ was superior to the other doses in measurements (Fig. 2a-e), we selected $30 \mathrm{mg} /$ 

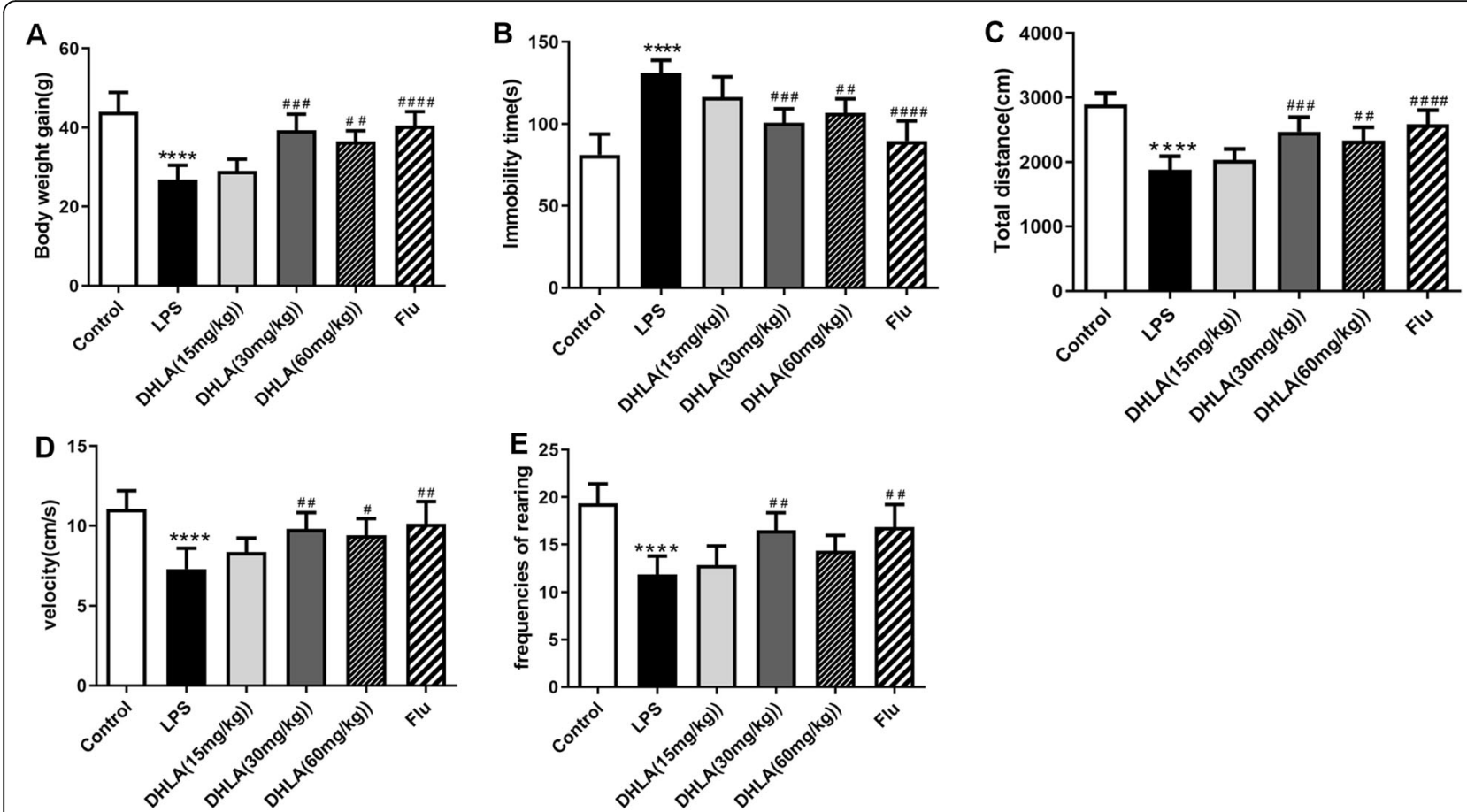

Fig. 2 Effect of different doses of DHLA on treatment of sickness behavior rats. a Effect of DHLA and Flu on body weight changes. b-e Depression-like behaviors were assessed by forced swimming test $(\mathbf{b})$ and open field test $(\mathbf{c}-\mathbf{e})$. The data were expressed as means \pm SEM $(n=6)$.

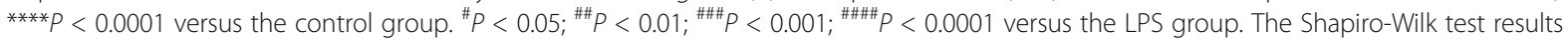
showed that all the data are normally distributed $(p>0.05)$

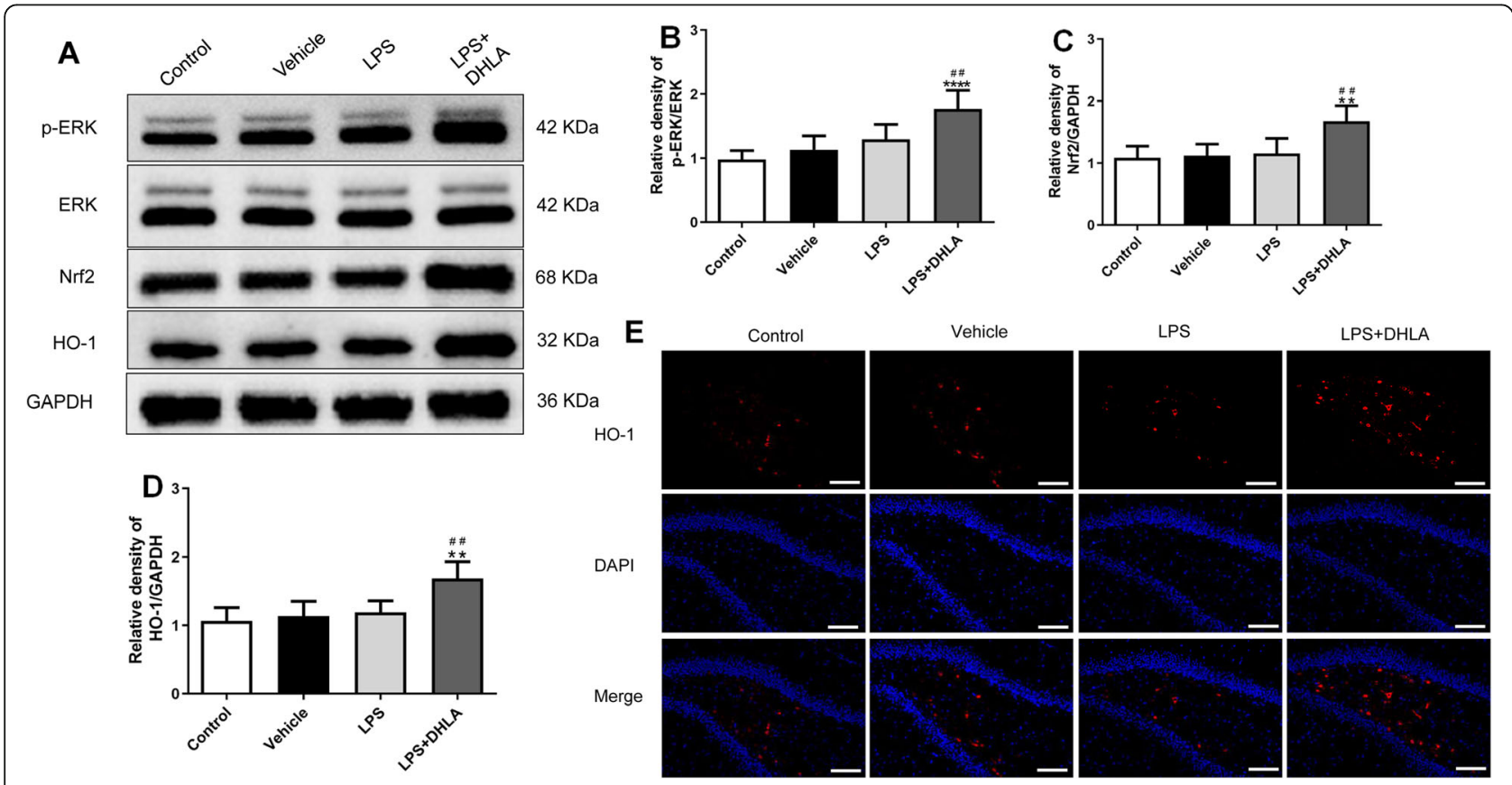

Fig. 3 Effect of DHLA on ERK/Nrf2/HO-1 signaling pathway in rats. a Representative Western blot bands in the hippocampal region. b-d Statistical graphs of relative protein expression of p-ERK/ERK (b), Nrf2 (c), and HO-1 (d). The data were expressed as means \pm SEM $(n=6)$. e Representative images of immunofluorescence assays of $\mathrm{HO}-1$ in the hippocampal. Scale bars represent $50 \mu \mathrm{m}$. The data were expressed as means \pm SEM $(n=3)$. ${ }^{* *} P<0.01 ;{ }^{* * *} P<0.0001$ versus the control group. ${ }^{\# \#} P<0.01$ versus the LPS group. The Shapiro-Wilk test results showed that all the data are normally distributed $(p>0.05)$ 
$\mathrm{kg}$ as the optimal dose of DHLA and used it in the following experiments.

\section{DHLA inhibited the activation of microglia induced by LPS}

To measure the number of microglia cells, immunofluorescence staining was performed by using the microglia-specific marker, IBA-1. As shown in Additional file 3: Fig. S3 A-B, rats exposed to LPS showed more number microglia as compared to the control group $(F(4,25)=22.16, p<0.001)$. However, treatment with DHLA $(p<0.01)$ decreased the microglia cell number as compared to the LPS group.

\section{DHLA reversed the LPS-induced sickness behavior through ERK/Nrf2/HO-1/ROS/NLRP3-dependent inflammation pathway}

Western blot and immunofluorescence staining were used to test the expression of ERK/Nrf2/HO-1/ROS/ NLRP3 signaling pathway in response to DHLA against LPS-induced sickness behavior in rats.

As shown in Fig. 3a-d, a statistically significant difference between groups for p-ERK $(F(3,20)=13.97, p<$ $0.0001)$, Nrf2 $(F(3,20)=9.48, p<0.0001)$, and HO-1 $(F(3,20)=10.90, p<0.0001)$ was determined by one-way ANOVA. Tukey's post hoc analysis revealed that expression levels of p-ERK $(p<0.01)$, Nrf2 $(p<0.01)$, and HO- $1(p<0.01)$ were significantly higher in DHLA-treated rats as compared to the LPS groups. Immunofluorescence staining revealed that the expression of HO-1 in the DHLA group was increased than that in the control group (Fig. 3e). As shown in Fig. 4a-d, a statistically significant difference in $\operatorname{NLRP3}(F(3,20)=21.62, p<$ $0.0001)$, caspase- $1(F(3,20)=17.37, p<0.0001)$, and IL$1 \beta(F(3,20)=11.93, p<0.0001)$ between the groups as determined by one-way ANOVA. Tukey's post hoc analysis revealed that the expression levels of NLRP3 $(p<$ $0.0001)$, caspase- $1(p<0.0001)$, and IL-1 $\beta(p<0.001)$ were significantly higher in LPS rats as compared to the control groups. However, these effects were significantly reversed by the treatment with DHLA (NLRP3, $p<0.01$; caspase- $1, p<0.01$; IL-1 $\beta, p<0.05$ ).

To verify the effect of DHLA alone on ERK/Nrf2/HO1/NLRP3 signaling pathway, we designed another four groups. As shown in Additional file 2: Fig. S2, a statistically significant difference between groups for p-ERK $(F(3,20)=30.09, p<0.0001), \operatorname{Nrf} 2(F(3,20)=10.57, p<$ $0.0001)$, HO-1 $(F(3,20)=12.03, p<0.0001)$, NLRP3 $(F(3,20)=28.55, p<0.0001)$, and $\operatorname{IL}-1 \beta(F(3,20)=16.58$, $p<0.0001)$ was determined by one-way ANOVA. Tukey's post hoc analysis revealed that expression levels of p-ERK $(p<0.01)$, Nrf2 $(p<0.01)$, and HO-1 $(p<$ 0.01 ) were significantly higher in DHLA alone group as compared to the LPS groups, and the expression levels

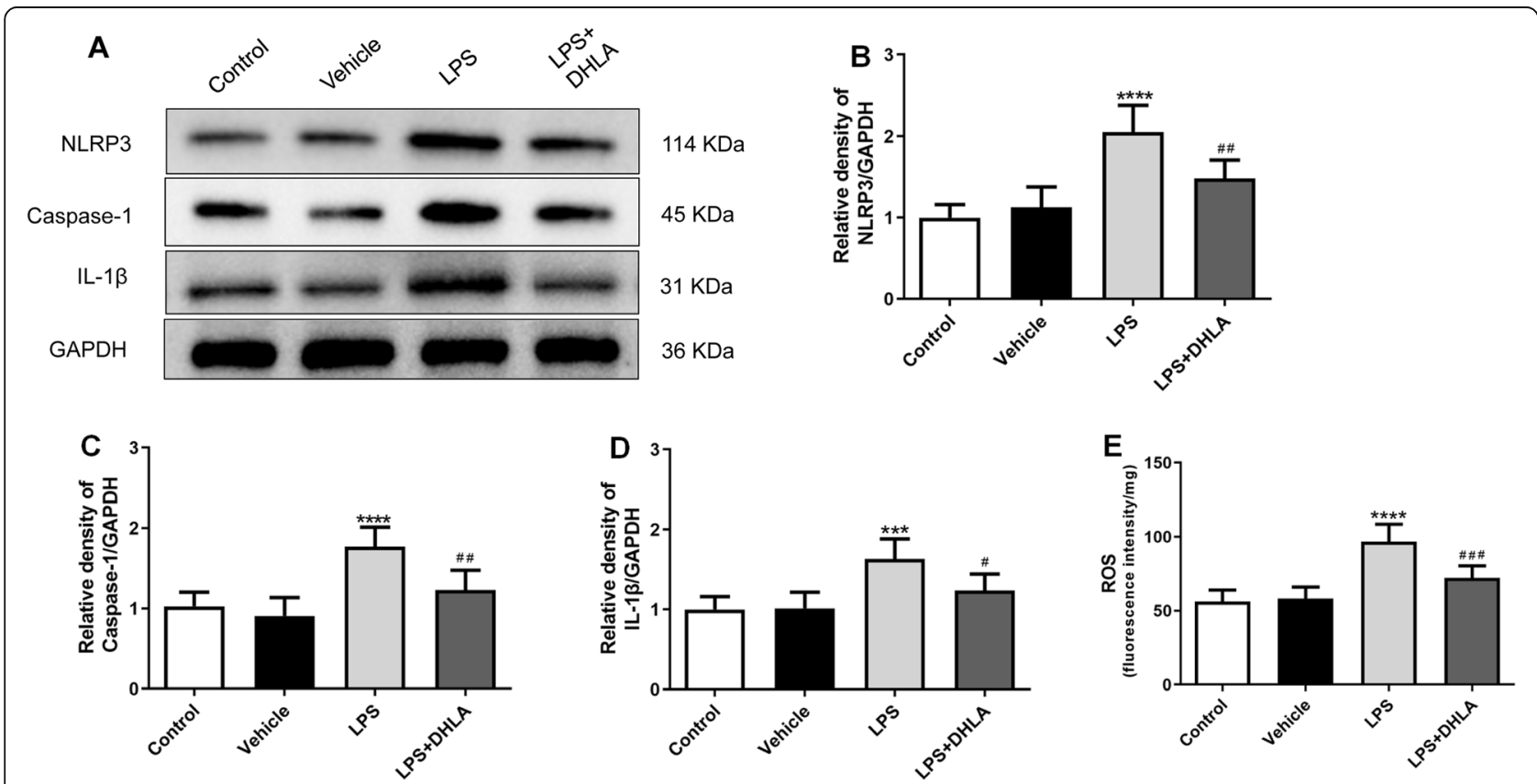

Fig. 4 Effect of DHLA on ROS/NLRP3/caspase-1/LL-1 $\beta$ signaling pathway in rats. a Representative Western blot bands in the hippocampal region. b-d Statistical graphs of relative protein expression of NLRP3 (b), caspase-1 (c), and IL-1 3 (d). e ROS expression in the hippocampal. The data

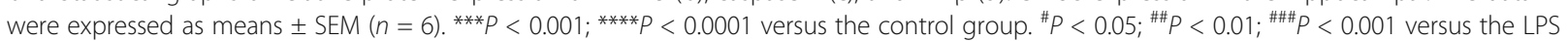
group. The Shapiro-Wilk test results showed that all the data are normally distributed $(p>0.05)$ 
of NLRP3 $(p<0.01)$ and IL-1 $\beta(p<0.05)$ were significantly lower in DHLA alone group as compared to the LPS groups.

The concentration of intracellular ROS was evaluated by the changes in DCF fluorescence intensity using flow cytometry. As shown in Fig. 4e, the ROS expression was distinctly higher in the LPS rats as compared to the control group $(F(3,20)=25.80, p<0.0001)$. Conversely, the intracellular ROS level induced by LPS was markedly reversed by DHLA $(p<0.001)$.

Bodyweight gain and behavioral tests, including OFT and FST, were performed to further investigate the effects of DHLA on LPS-induced sickness behavior in rats. As shown in Additional file 1: Fig. S1A, rats exposed to LPS showed less body weight gain than the control group $(F(5,30)=20.84, p<0.0001)$. However, treatment with DHLA (30 mg/kg, $p<0.01$ ) improved the bodyweight gain as compared to the LPS group. Additional file 1: Fig. S1B showed that rats exposed to LPS showed more immobility time in FST as compared to the control group $(F(5,30)=27.11, p<0.0001)$. On the other hand, compared to the LPS group, DHLA $(30 \mathrm{mg} / \mathrm{kg}, p<$ 0.001) treatment markedly decreased the immobility time in FST. As shown in Additional file 1: Fig. S1C-E, the total distance, total velocity, and rearing frequencies were significantly decreased in the LPS group as compared to the control group (total distance $(F(5,30)=$ $30.48, p<0.0001)$; velocity $(F(5,30)=12.16, p<0.0001)$; rearing frequencies $(F(5,30)=15.22, p<0.0001)$. Compared to the LPS group, the total distance, total velocity, and rearing frequencies in the DHLA $(30 \mathrm{mg} / \mathrm{kg}$, all $p<$ 0.01 ) group were significantly increased.

\section{Blockade of ERK abolished the preventive effect and anti-} inflammation effect of DHLA

Previous studies have suggested that the ERK pathway is related to the regulation of Nrf2 [29]. PD98059 (an ERK pathway inhibitor) was administered in LPS-induced sickness behavior rats $1 \mathrm{~h}$ before the administration of DHLA to investigate whether DHLA upregulated the expression of Nrf2/HO-1 in LPS-induced rats via the ERK pathway.

As shown in Fig. 5a, rats exposed to DHLA showed higher body weight gain as compared to the LPS group $(F(5,30)=22.61, p<0.0001)$. However, treatment with PD98059 $(p<0.01)$ decreased body weight gain than the DHLA group.

As shown in Fig. 5b-e, the DHLA administration greatly attenuated the depression-like behavior in LPSinduced rats. As shown in Fig. 5b, rats exposed to DHLA

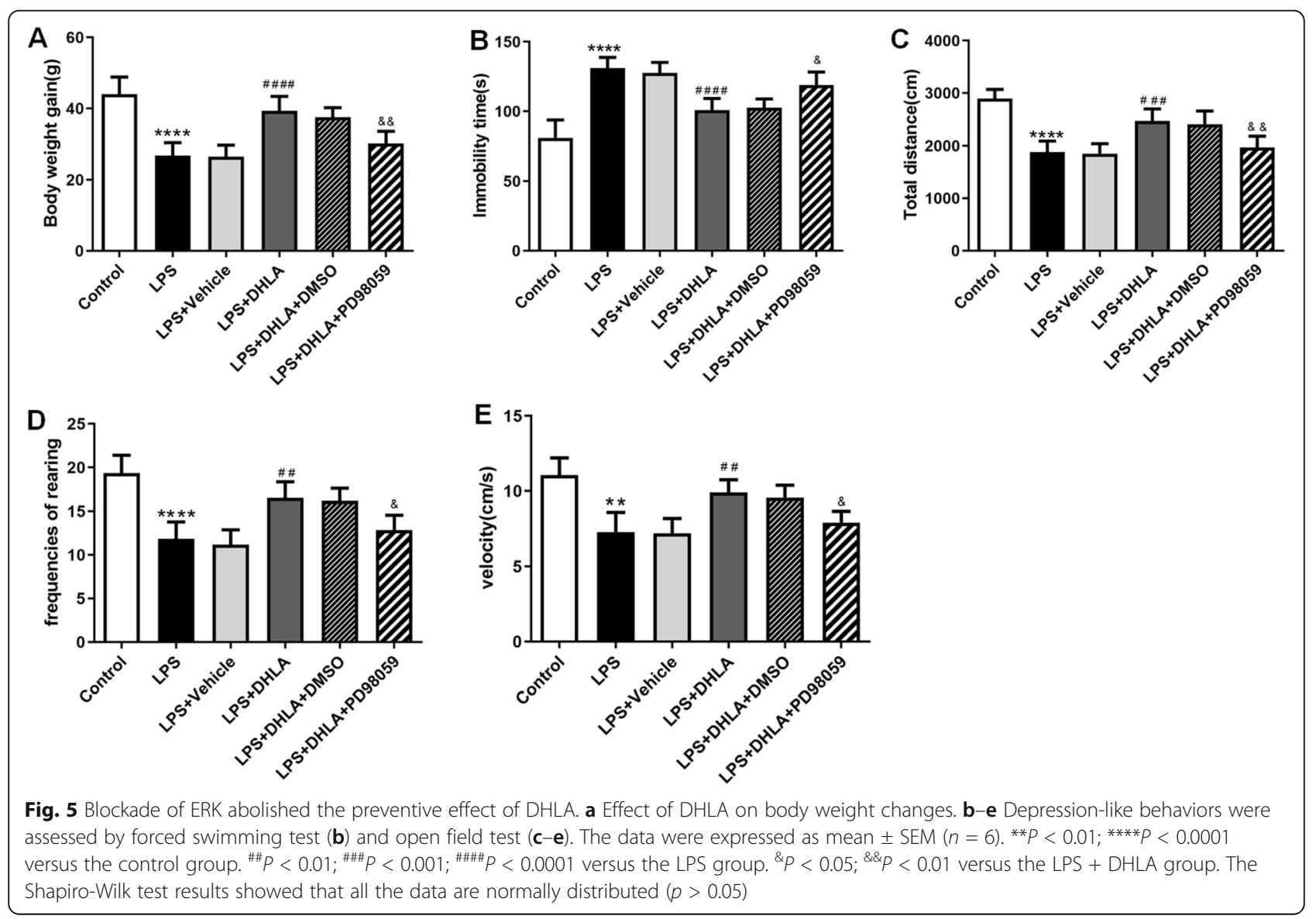


showed less immobility time in FST as compared to the LPS group $(F(5,30)=26.79, p<0.0001)$, whereas compared to the DHLA group, the PD98059 $(p<0.05)$ treatment markedly increased the immobility time in FST. As shown in Fig. $5 \mathrm{c}-\mathrm{e}$, the total distance, rearing frequencies, and total velocity were significantly increased in the DHLA group as compared to the LPS group (total distance $F(5,30)=22.78, p<0.0001$; rearing frequencies $F(5,30)=18.71, p<0.0001$; velocity $F(5,30)=15.17, p<$ $0.0001)$. However, compared to the DHLA group, the total distance in the PD98059 $(p<0.01)$ group and rearing frequencies and total velocity in the PD98059 (both $p<0.05)$ group were significantly decreased. These data indicated that the preventive effect of DHLA in the LPSinduced rats was blocked by PD98059.

As shown in Fig. 6a-f, the results showed a statistically significant difference between the study groups as determined by one-way ANOVA with respect to $\operatorname{Nrf2}(F(5$, $30)=35.07, p<0.0001)$, HO-1 $(F(5,30)=25.19, p<$ $0.0001), \operatorname{NLRP3}(F(5,30)=50.79, p<0.0001)$, caspase -1 $(F(5,30)=15.64, p<0.0001)$, and IL-1 $(F(5,30)=34.71$, $p<0.0001)$. Tukey's post hoc analysis revealed that expression levels of Nrf2 $(p<0.0001)$ and HO-1 $(p<$ 0.0001 ) were significantly higher in DHLA rats as compared to the LPS groups. In addition, the expression of NLRP3 $(p<0.0001)$, caspase- $1(p<0.01)$, and IL-1 $\beta(p<$ $0.0001)$ was significantly lower in DHLA rats as compared to the LPS groups. However, inhibition of ERK with PD98059 abolished the effects of DHLA, which led to decreased Nrf2 $(p<0.01)$ and HO-1 $(p<$ $0.01)$, while increased in the inflammation-related proteins, such as NLRP3 $(p<0.01)$, caspase-1 $(p<0.05)$, and IL-1 $\beta(p<0.01)$ as compared to the DHLA group.

As shown in Fig. 6g, the ROS expression was distinctly lower in the DHLA group as compared to the LPS group $(F(5,30)=23.09, p<0.0001)$. Conversely, the ROS level induced by DHLA was markedly ameliorated by PD98059 $(p<0.01)$.

Blockade of Nrf2 abolished the preventive effect and antiinflammation effect of DHLA

Bodyweight gain and behavioral tests showed that AAVNrf2-siRNA completely abolished the treatment effects of DHLA in the LPS-induced sickness behavior rats.

As shown in Fig. 7a, rats exposed to DHLA showed higher body weight gain as compared to the LPS group $(F(5,30)=17.46, p<0.0001)$. However, treatment with AAV-Nrf2-siRNA $(p<0.05)$ decreased body weight gain as compared to the DHLA group.

As shown in Fig. 7b-e, the DHLA administration greatly attenuated the sickness behaviors observed in LPS-induced rats. As shown in Fig. 7b, rats exposed to DHLA showed less immobility time in FST as compared to the LPS group $(F(5,30)=24.1, p<0.0001)$. On the
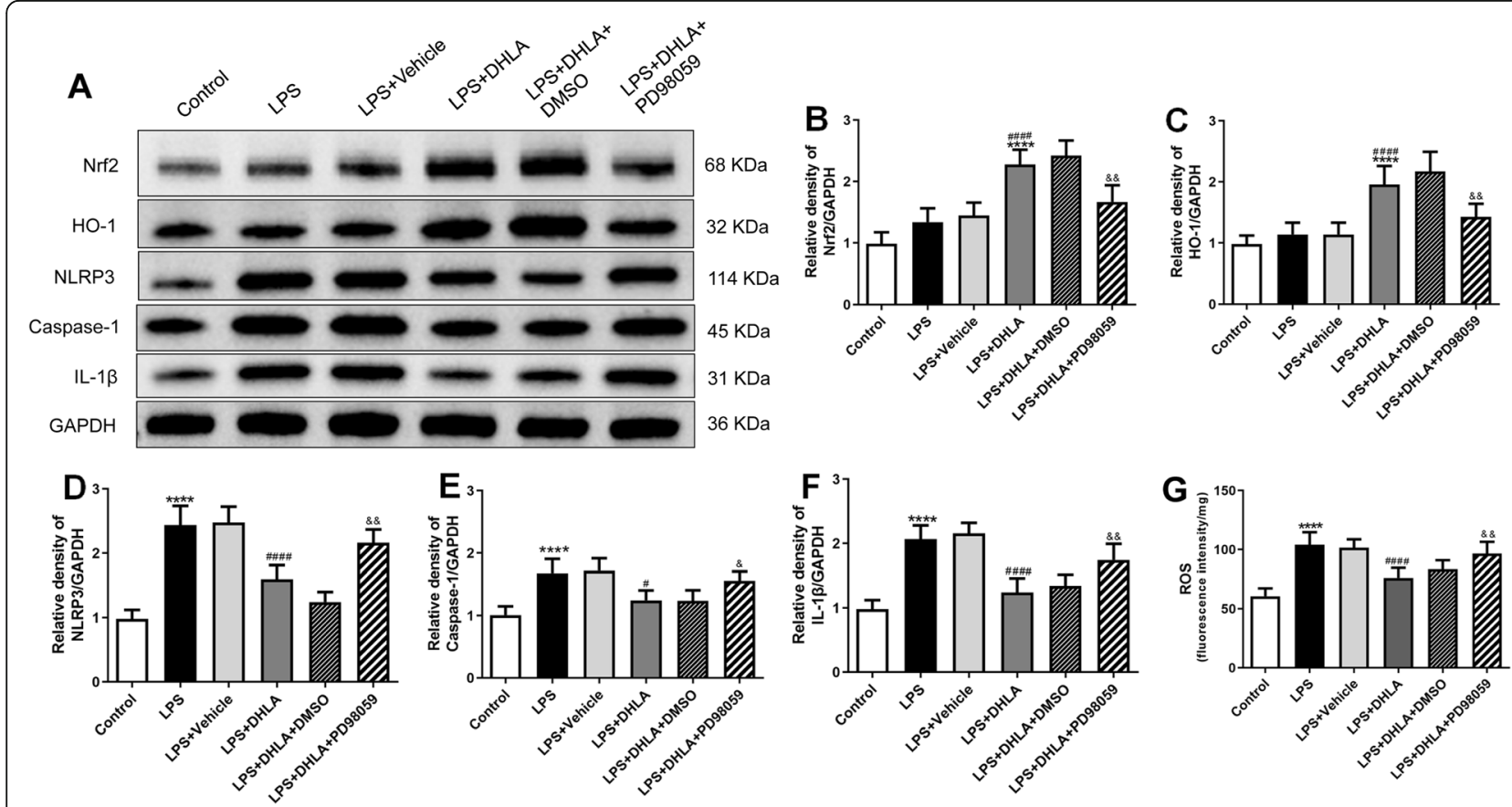

Fig. 6 Blockade of ERK abolished the anti-Inflammation effect of DHLA. a Representative Western blot bands in the hippocampal region. b-f Statistical graphs of relative protein expression of Nrf2 (b), HO-1 (c), NLRP3 (d), caspase-1 (e), and IL-1 3 (f). g ROS expression in the hippocampal. The data were expressed as means \pm SEM $(n=6)$. ${ }^{* * *} P<0.0001$ versus the control group. ${ }^{\#} P<0.05$; ${ }^{\# \# \# \# ~} P<0.0001$ versus the LPS group. ${ }^{*} P<$ $0.05 ;{ }^{\& \&} P<0.01$ versus the LPS + DHLA group. The Shapiro-Wilk test results showed that all the data are normally distributed ( $\left.p>0.05\right)$ 


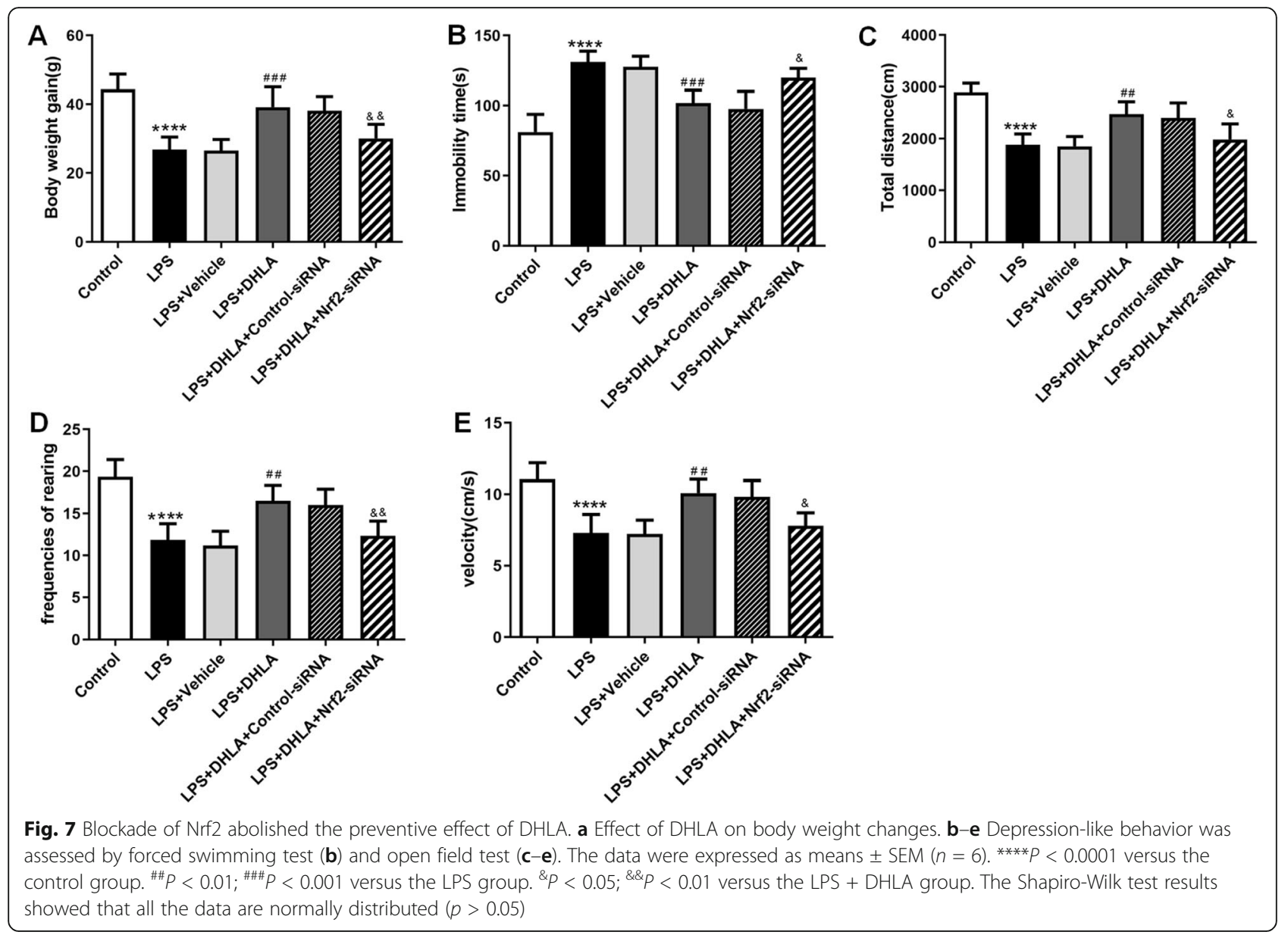

other hand, compared to the DHLA group, the AAVNrf2-siRNA $(p<0.05)$ treatment markedly increased the immobility time in FST. As shown in Fig. 7c-e, the total distance, rearing frequencies, and total velocity were significantly increased in the DHLA group that in the LPS group (total distance $F(5,30)=17.84, p<0.0001$; rearing frequencies $F(5,30)=17.87, p<0.0001$; velocity $F(5,30)$ $=13.86, p<0.0001)$. However, compared to the DHLA group, the total distance, the rearing frequencies, and total velocity in the DHLA $(p<0.05, p<0.01, p<0.05$, respectively) group were significantly decreased. These data indicated that the preventive effect of DHLA in LPS-induced rats was blocked by AAV-Nrf2-siRNA.

As shown in Fig. 8a-e, the results showed a statistically significant difference between the study groups as determined by one-way ANOVA regarding Nrf2 $(F(5,30)$ $=11.77, p<0.0001)$, HO-1 $(F(5,30)=41.88, p<0.0001)$, NLRP3 $(F(5,30)=23.01, p<0.0001)$, caspase-1 $(F(5,30)$ $=20.75, p<0.0001)$, and $\operatorname{IL}-1 \beta(F(5,30)=25.14, p<$ $0.0001)$. Tukey's post hoc analysis revealed that the expression levels of Nrf2 $(p<0.01)$ and HO-1 $(p<0.0001)$ were significantly higher in DHLA rats as compared to that in the LPS groups. Moreover, the expression of
NLRP3 $(p<0.001)$, caspase-1 $(p<0.001)$, and IL-1 $\beta(p<$ $0.0001)$ was significantly lower in DHLA rats as compared to the LPS groups. However, inhibition of Nrf2 with AAV-Nrf2-siRNA abolished the effects of DHLA, which led to a decrease in Nrf2 $(p<0.05)$ and HO-1 ( $p$ $<0.01)$ expression and increase in the expression of inflammation-related proteins NLRP3 $(p<0.01)$, caspase-1 $(p<0.01)$, and IL-1 $\beta(p<0.001)$ as compared to the DHLA group.

As shown in Fig. 8g, the results showed that the ROS expression was distinctly lower in the DHLA group as compared to the LPS group $(F(5,30)=14.89, p<$ $0.0001)$. In contrast, the ROS level induced by DHLA was markedly ameliorated by AAV-Nrf2-siRNA $(p<$ 0.05).

\section{Discussion}

The present study revealed that the sickness behaviors induced by LPS in rats are reversed by DHLA treatment with the anti-depressant Flu and is associated with the upregulation of Nrf2 and HO-1. The inhibition of ERK abolished the preventive effect of DHLA, which was related to the low expression of $\mathrm{Nrf} 2$ and $\mathrm{HO}-1$ and 

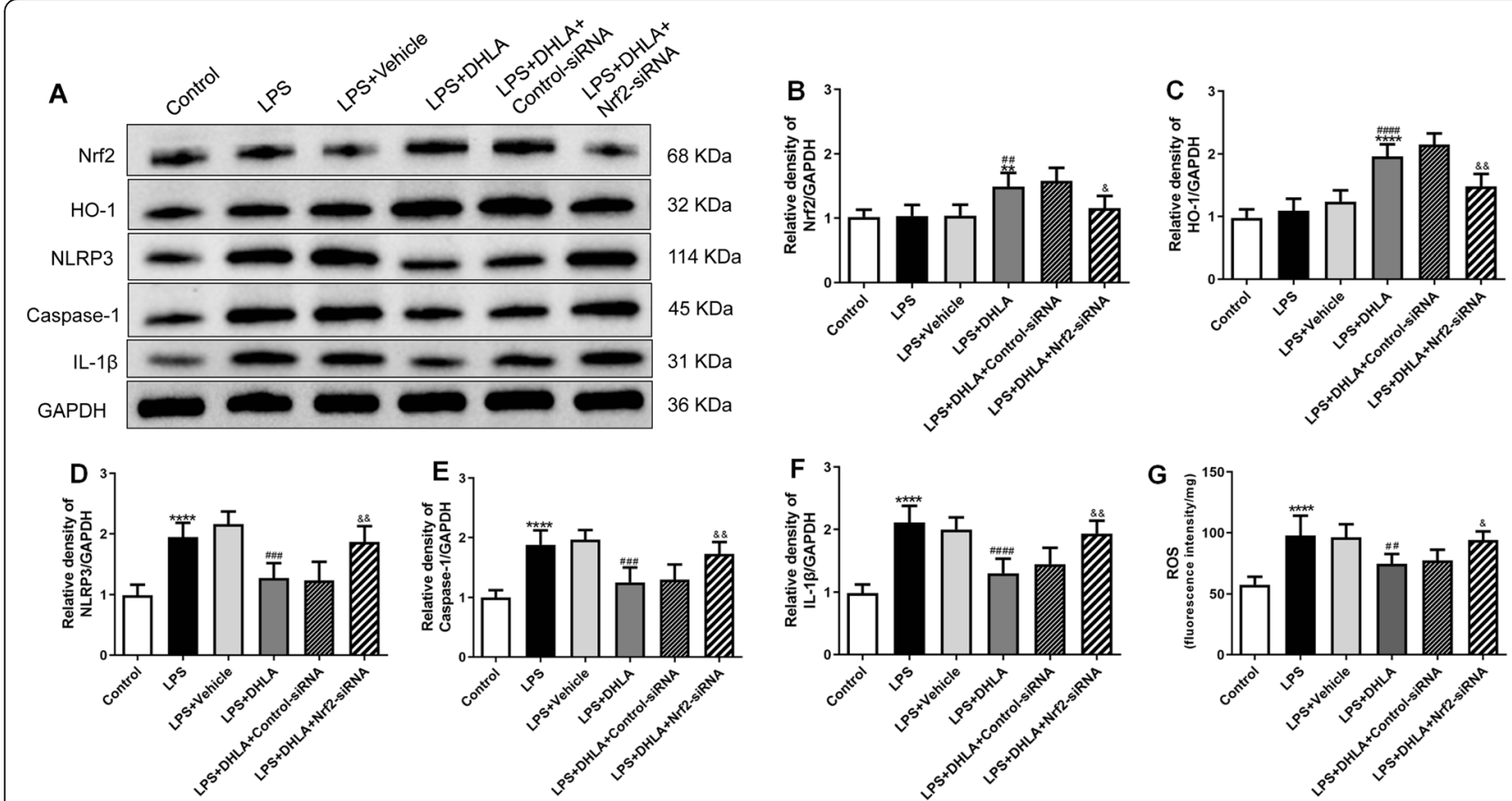

Fig. 8 Blockade of Nrf2 abolished the anti-Inflammation effect of DHLA. a Representative Western blot bands in the hippocampal region. b-f Statistical graphs of relative protein expression of Nrf2 (b), HO-1 (c), NLRP3 (d), caspase-1 (e), and IL-1 3 (f). $\mathbf{g}$ ROS expression in the hippocampal.

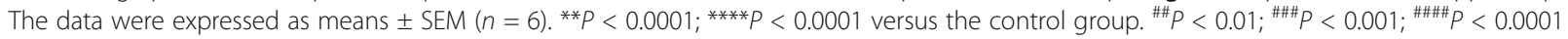
versus the LPS group. ${ }^{\&} P<0.05 ;{ }^{\&} \&<0.01$ versus the LPS + DHLA group. The Shapiro-Wilk test results showed that all the data are normally distributed $(p>0.05)$

upregulation of NLRP3, caspase-1, and IL-1ß. Moreover, the knockdown of Nrf2 using Nrf2 siRNA abolished the effect of the DHLA treatment effect similarly, which was associated with a decrease in $\mathrm{HO}-1$ and upregulation of NLRP3, caspase-1, and IL-1 $\beta$. Taken together, our findings suggested that DHLA protects against LPS-induced behavioral deficits and neuroinflammation through ERK/ Nrf2/HO-1/ROS/NLRP3 signaling pathway in rats.

Lipoic acid is a natural lipophilic antioxidant that has beneficial effects on heart diseases, diabetes, and neuroinflammatory disorders [30-33]. Lipoic acid occurs in two forms: $\alpha$-lipoic acid and DHLA [34]. DHLA is the reduced form of lipoic acid with a more specific and useful outcome than $\alpha$-lipoic acid [35]. DHLA is considered to have more antioxidant properties than LA, because DHLA has the properties of endogenous antioxidants regeneration and repairing oxidative damage [36]. Moreover, the neuroprotective effects of DHLA have been shown in several central nervous system diseases, such as ischemic stroke, traumatic brain injury. Recently, DHLA was indicated as an effective treatment for subarachnoid hemorrhage [19]. However, no study has been published, wherein DHLA was used as a therapeutic method for depression. To the best of our knowledge, this is the first study evaluating the preventive effect of DHLA on sickness behaviors in rats.
Herein, we first evaluated the effect of three different dosages of DHLA on LPS-induced sickness behavior in rats. In the current study, $30 \mathrm{mg} / \mathrm{kg}$ DHLA is the most effective dose for the treatment of sickness behaviors. In line with our results, a previous study has suggested that $30 \mathrm{mg} / \mathrm{kg}$ DHLA contributes to the neuroprotective effects [19]. Thus, this dose may be optimal for the treatment of nervous system disease.

LPS is a component of the outer cell wall of Gramnegative bacteria and is widely used to induce inflammation in the brain. Nrf2 is a major transcription factor that regulates cell antioxidant response. Nrf2 is transported to the nucleus under oxidative stress and binds to the antioxidant response elements (ARE) to regulate the expression of antioxidant enzymes such as $\mathrm{HO}-1$ [37]. In the previous study, the expression of the Nrf2systerm (Nrf2 and HO-1) in the rat brain was still increased 3 days after the LPS systemic injection, decreased by 7 days, and then gradually normalized after LPS [38]. Our results showed that the contents of Nrf2 and $\mathrm{HO}-1$ were nearly normalized in the hippocampus of rats after 14 days of LPS-induced. The temporal shift of Nrf2-systerm is likely caused by a prolonged acute phase of inflammation as a result of the higher dose of LPS. Consecutively, we found that DHLA treatment increased the expression of Nrf2 and HO-1, which was 
abrogated when Nrf2 was downregulated by AAV-Nrf2siRNA.

Previous studies indicated that upregulated HO-1 expression exerts a protective effect against increased levels of ROS [39]. In the present study, the ROS level increased significantly in the LPS-induced groups as compared to the control groups but was restored in the DHLA treatment group.

Therefore, we speculated that DHLA regulates the ROS expression by activating the Nrf2/HO-1 signaling pathway. However, the mechanisms underlying the DHLA-mediated and Nrf2 activation after LPS-induced sickness behavior have not yet been elucidated. Previous studies have shown that Nrf2 activation is regulated by the MAPK/ERK pathway [40]. We postulated that treatment with DHLA might regulate Nrf2 expression via the ERK pathway in rats. To further confirm our hypothesis, we used an inhibitor of ERK to test the expression of Nrf2 after DHLA treatment. Previous studies have shown that LPS can significantly increase phosphorylation levels of ERK in the hippocampus of mice [41, 42]. At the same time, one study demonstrated that LPS did not affect ERK phosphorylation in the hippocampus of mice [43]. In our study, we observed that LPS did not affect the phosphorylation of ERK in the hippocampus. The different expression of p-ERK induced by LPS may be related to the dose of LPS and stimulation time of LPS. Then, we found that DHLA promoted the expression of ERK and Nrf2. Strikingly, the expression of Nrf2 could be inhibited by the ERK inhibitor PD98059. Thus, we speculated that DHLA activates Nrf2 through the ERK signaling pathway. These findings indicated that DHLA improves the sickness behaviors induced by LPS in rats, primarily through the ERK/Nrf2/HO-1/ROS pathway.

In recent years, many studies have shown that the pathogenesis and progression of depression are related to the overreaction of inflammation and immune responses $[4,44]$. The inflammasome is an inducer of immune response with the function of identifying and targeting multiple pathogens [45]. Hitherto, several forms of inflammasomes have been reported, including NLRP1, NLRP2, NLRP3, and NLRC4 inflammasomes [46]. Among these, NLRP3 inflammasome is involved in the onset and progression of several diseases [47]. It is composed of NLRP3 protein, adaptor protein apoptosisrelated speckles (ASC), and procaspase-1 $[48,49]$. The activation of NLRP3 inflammasome triggers the transformation of procaspase- 1 to caspase- 1 and catalyzes the exudation of mature IL-1 $\beta$ and IL-18 from pro-IL-1 $\beta$ and pro-IL-18, causing an inflammatory response [50, 51]. Previous studies showed that ROS, especially from mitochondria, activated the NLRP3 inflammasome [52, 53]. The current findings proposed that the expression of ROS, NLRP3, caspase-1, and IL-1 $\beta$ in the LPS group was increased as compared to that in the control groups; however, after the administration of DHLA, the level of NLRP3, caspase- 1 , and IL-1 $\beta$ decreased markedly.

Microglia are the main immune cells in the CNS and are involved in neuroprotection and immunosurveillance through the regulation of various pro-inflammatory cytokines [54]. Some studies have shown that microglia is activated in neuroinflammation, contributing to the development of depression $[55,56]$. Excessive activation of microglia promotes the production of pro-inflammatory factors, such as IL-1 $\beta$, and damages synaptic function. Our results showed that the number of microglia was significantly increased in the LPS-induced groups. However, DHLA evidently inhibited the increase of microglial cells. Therefore, we speculated that inhibition of microglia activation may be related to the anti-inflammatory effect of DHLA.

Furthermore, these results supported DHLA treatment to attenuate the oxidative stress-related neuroinflammation and microglial activation for LPS-induced sickness behavior. Nevertheless, our study is not without limitations, first, the downstream mechanism of Nrf2 to NLRP3 is complex; hence, we targeted only one portion of the pathway. Thus, the probability of other factors involved in DHLA treatment needs further investigation. Second, the protective effects of DHLA might be observed in other cell types such as neurons or astrocytes, and hence, these factors also need to be explored further. Third, in our study, we used LPS-injected rats as our animal model, and this model is widely used as a sickness behavior model and seems to be for a limited condition of depression.

\section{Conclusions}

In conclusion, our study suggests that DHLA has a preventive effect on sickness behaviors in rats with LPSinduced, and these effects are exerted via regulation of Nrf2/HO-1/ROS/NLRP3 signaling pathway and microglial activation. These results provide a theoretical basis for DHLA as a promising method in the treatment of depression.

\section{Supplementary information}

Supplementary information accompanies this paper at https://doi.org/10. 1186/s12974-020-01836-y.

Additional file 1: Figure S1. Effect of optimal dose of DHLA on treatment of sickness behavior rats. a Effect of DHLA on body weight changes. b-e Depression-like behavior was assessed by forced swimming test (b) and open field test $(\mathbf{c}, \mathbf{d}, \mathbf{e})$. The data were expressed as means \pm SEM (n=6). ${ }^{* * * *} P<0.0001$, versus the control group. ${ }^{\# \#} P<0.01$; ${ }^{\# \#} P<$ 0.001 versus the LPS group. The Shapiro-Wilk test results showed that all the data are normally distributed $(p>0.05)$. 
Additional file 2: Figure S2. Effect of DHLA on ERK/Nrf2/HO-1/NLRP3/ IL-1 $\beta$ signaling pathway in rats. a-b Representative Western blot bands in the hippocampal region. $\mathbf{c - g}$ Statistical graphs of relative protein expression of p-ERK/ERK (c), Nrf2 (d), HO-1 (e), NLRP3 (f), IL-1 $\beta$ (g). The data were expressed as means \pm SEM $(n=6)$. ${ }^{*} P<0.01 ;{ }^{* * *} P<0.001$; ${ }^{* * * *} P<0.0001$, versus the control group. ${ }^{\#} P<0.05 ;{ }^{\# \#} P<0.01$, versus the LPS group. The Shapiro-Wilk test results showed that all the data are normally distributed $(p>0.05)$.

Additional file 3: Figure S3. Effect of optimal dose of DHLA prevents LPS-induced increase of the microglial number. a Representative images of immunofluorescence assays of Iba1 in the hippocampus. Six micrographs from three rats per group were analyzed. b DHLA blocked the increased lba-1 signal intensity induced by LPS. Scale bars represent $50 \mu \mathrm{m}$. The data were expressed as means $\pm \operatorname{SEM}(n=6)$. ${ }^{* *} P<0.001$, versus the

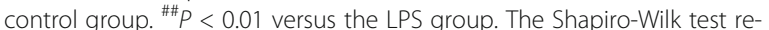
sults showed that all the data are normally distributed $(p>0.05)$.

\section{Abbreviations}

DHLA: Dihydrolipoic acid; FST: Forced swim test; HO-1: Heme oxygenase-1; LPS: Lipopolysaccharide; NLRP3: Nod-like receptor pyrin containing 3 inflammasome; Nrf2: Nuclear factor erythroid 2-like; OFT: Open field test; ROS: Reactive oxygen species

\section{Acknowledgements}

We wish to thank Jun Wang for his excellent technical assistance.

\section{Authors' contributions}

Authors G-HW and H-TB designed the study and wrote the protocol. Authors $H-T B, J-J H, L L, Y-G Z, Y-Y W$, and HW performed the experiments and analyzed the data. Author $\mathrm{H}-\mathrm{TB}, \mathrm{J}-J \mathrm{H}$, and LX managed the literature searches and figure drawing. Authors H-TB wrote the manuscript. L-X, H-LW, and G-HW revised the manuscript. All authors contributed to and have approved the final manuscript.

\section{Funding}

This research was conducted with funding by grants from the National Natural Science Foundation of China (81571325, 81871072, and 81301012) and the Medical Science Advancement Program of Wuhan University (NO.TFLC2018001)

\section{Availability of data and materials}

All the necessary data are included within the article. Further data will be shared by request.

\section{Ethics approval and consent to participate}

All procedures involving animals were approved and carried out according to the guidelines of the Institutional Animals Care Committee of Renmin Hospital of Wuhan University.

\section{Consent for publication}

Not applicable

\section{Competing interests}

The authors declare that they have no competing interests.

Received: 13 January 2020 Accepted: 3 May 2020

Published online: 25 May 2020

\section{References}

1. Sarris J, Murphy J, Mischoulon D, Papakostas Gl, Fava M, Berk M, et al. Adjunctive nutraceuticals for depression: a systematic review and metaanalyses. Am J Psychiatry. 2016;173:575-87.

2. Enache D, Pariante CM, Mondelli V. Markers of central inflammation in major depressive disorder: a systematic review and meta-analysis of studies examining cerebrospinal fluid, positron emission tomography and postmortem brain tissue. Brain Behav Immun. 2019;81:24-40.

3. Inserra A, Mastronardi CA, Rogers G, Licinio J, Wong ML. Neuroimmunomodulation in major depressive disorder: focus on caspase 1, inducible nitric oxide synthase, and interferon-gamma. Mol Neurobiol. 2019; 56:4288-305.
4. Ironside M, Admon R, Maddox SA, Mehta M, Douglas S, Olson DP, et al. Inflammation and depressive phenotypes: evidence from medical records from over 12000 patients and brain morphology. Psychol Med. 2019:1-9.

5. Leu WJ, Chen JC, Guh JH. Extract from plectranthus amboinicus inhibit maturation and release of interleukin 1 beta through inhibition of NF-kappaB nuclear translocation and nlrp3 inflammasome activation. Front Pharmacol. 2019;10:573.

6. Kuwar R, Rolfe A, Di L, Xu H, He L, Jiang Y, et al. A novel small molecular NLRP3 inflammasome inhibitor alleviates neuroinflammatory response following traumatic brain injury. J Neuroinflammation. 2019;16:81.

7. Hill RL, Singh IN, Wang JA, Hall ED. Time courses of post-injury mitochondrial oxidative damage and respiratory dysfunction and neuronal cytoskeletal degradation in a rat model of focal traumatic brain injury. Neurochem Int. 2017;111:45-56.

8. Rizwan H, Pal S, Sabnam S, Pal A. High glucose augments ROS generation regulates mitochondrial dysfunction and apoptosis via stress signalling cascades in keratinocytes. Life Sci. 2020;241:117148.

9. Arioz BI, Tastan B, Tarakcioglu E, Tufekci KU, Olcum M, Ersoy N, et al. Melatonin attenuates LPS-induced acute depressive-like behaviors and microglial NLRP3 inflammasome activation through the SIRT1/Nrf2 pathway. Front Immunol. 2019;10:1511.

10. Harper SN, Leidig PD, Hughes FM Jr, Jin H, Purves JT. Calcium pyrophosphate and monosodium urate activate the NLRP3 inflammasome within bladder urothelium via reactive oxygen species and TXNIP. Res Rep Urol. 2019;11:319-25.

11. Bollinger $\mathrm{L}$, , Wohleb ES. The formative role of microglia in stress-induced synaptic deficits and associated behavioral consequences. Neurosci Lett. 2019;711:134369.

12. Robledinos-Anton N, Fernandez-Gines R, Manda G, Cuadrado A. Activators and inhibitors of NRF2: a review of their potential for clinical development. Oxidative Med Cell Longev. 2019;2019:9372182.

13. Zhu X, Guo F, Tang H, Huang C, Xie G, Huang T, et al. Islet transplantation attenuating testicular injury in type 1 diabetic rats is associated with suppression of oxidative stress and inflammation via Nrf-2/HO-1 and NFkappaB pathways. J Diabetes Res. 2019;2019:8712492.

14. Sun GY, Li R, Cui J, Hannink M, Gu Z, Fritsche KL, et al. Withania somnifera and its withanolides attenuate oxidative and inflammatory responses and up-regulate antioxidant responses in BV-2 microglial cells. NeuroMolecular Med. 2016;18:241-52.

15. Taira J, Ogi T. Induction of antioxidant protein HO-1 through Nrf2-ARE signaling due to pteryxin in Peucedanum japonicum Thunb in RAW264.7 macrophage cells. Antioxidants (Basel). 2019, 8.

16. Aladaileh SH, Hussein OE, Abukhalil MH, Saghir SAM, Bin-Jumah M, Alfwuaires MA, et al. Formononetin upregulates Nrf2/HO-1 signaling and prevents oxidative stress, inflammation, and kidney injury in methotrexateinduced rats. Antioxidants (Basel). 2019, 8.

17. Liu WH, Shi LS, Chung MC, Chang TC, Lee SY. Antcamphin M inhibits TLR4mediated inflammatory responses by upregulating the $\mathrm{Nrf} 2 / \mathrm{HO}-1$ pathway and suppressing the NLRP3 inflammasome pathway in macrophages. Am J Chin Med. 2019:1-16.

18. Lou Y, Guo Z, Zhu Y, Kong M, Zhang R, Lu L, et al. Houttuynia cordata Thunb. and its bioactive compound 2-undecanone significantly suppress benzo(a)pyrene-induced lung tumorigenesis by activating the Nrf2-HO-1/ NQO-1 signaling pathway. J Exp Clin Cancer Res. 2019:38-242.

19. Zhou K, Enkhjargal B, Xie Z, Sun C, Wu L, Malaguit J, et al. Dihydrolipoic acid inhibits lysosomal rupture and NLRP3 through lysosome-associated membrane protein-1/calcium/calmodulin-dependent protein kinase II/TAK1 pathways after subarachnoid hemorrhage in rat. Stroke. 2018;49:175-83.

20. Jiang P, Guo Y, Dang R, Yang M, Liao D, Li H, et al. Salvianolic acid B protects against lipopolysaccharide-induced behavioral deficits and neuroinflammatory response: involvement of autophagy and NLRP3 inflammasome. J Neuroinflammation. 2017;14:239.

21. Jin Q, Ju J, Xu L, Liu Y, Li Z, Fu Y, et al. Estradiol postconditioning relieves ischemia/reperfusion injury in axial skin flaps of rats, inhibits apoptosis and alters the MKP-1/ERK pathway. Mol Med Rep. 2017;16:1472-8.

22. Sumaya IC, Bailey D, Catlett SL. Differential effects of a short-term high-fat diet in an animal model of depression in rats treated with the $5-\mathrm{HT} 3$ receptor antagonist, ondansetron, the 5-HT3 receptor agonist, 2-methyl-5HT, and the SSRI, fluoxetine. Pharmacol Biochem Behav. 2016;144:78-84.

23. Bai J, Yu XJ, Liu KL, Wang FF, Jing GX, Li HB, et al. Central administration of tert-butylhydroquinone attenuates hypertension via regulating Nrf2 
signaling in the hypothalamic paraventricular nucleus of hypertensive rats. Toxicol Appl Pharmacol. 2017;333:100-9.

24. McKee AG, Loscher JS, O'Sullivan NC, Chadderton N, Palfi A, Batti L, et al. AAV-mediated chronic over-expression of SNAP-25 in adult rat dorsal hippocampus impairs memory-associated synaptic plasticity. J Neurochem. 2010;112:991-1004.

25. Wei Y, Wang G, Wang H, He J, Zhang N, Wu Z, et al. Sex-dependent impact of different degrees of maternal separation experience on OFT behavioral performances after adult chronic unpredictable mild stress exposure in rats. Physiol Behav. 2018;194:153-61.

26. Zhang Y, Sun J, Zhu S, Xu T, Lu J, Han H, et al. The role of rhynchophylline in alleviating early brain injury following subarachnoid hemorrhage in rats. Brain Res. 1631;2016:92-100.

27. Xiang D, Xiao J, Fu L, Yao L, Wan Q, Xiao L, et al. DNA methylation of the Tacr2 gene in a CUMS model of depression. Behav Brain Res. 2019;365:1039.

28. Wang YL, Han QQ, Gong WQ, Pan DH, Wang LZ, Hu W, et al. Microglial activation mediates chronic mild stress-induced depressive- and anxiety-like behavior in adult rats. J Neuroinflammation. 2018;15:21.

29. Qi Z, Ci X, Huang J, Liu Q, Yu Q, Zhou J, et al. Asiatic acid enhances Nrf2 signaling to protect HepG2 cells from oxidative damage through Akt and ERK activation. Biomed Pharmacother. 2017;88:252-9.

30. Paradells-Navarro S, Benlloch-Navarro MS, Almansa Frias Ml, Garcia-Esparza MA, Broccoli V, Miranda M, et al. Neuroprotection of brain cells by lipoic acid treatment after cellular stress. ACS Chem Neurosci. 2017:8:569-77.

31. Li YH, He Q, Yu JZ, Liu CY, Feng L, Chai Z, et al. Lipoic acid protects dopaminergic neurons in LPS-induced Parkinson's disease model. Metab Brain Dis. 2015;30:1217-26.

32. Miao Y, Ren J, Jiang L, Liu J, Jiang B, Zhang $X$. alpha-Lipoic acid attenuates obesity-associated hippocampal neuroinflammation and increases the levels of brain-derived neurotrophic factor in ovariectomized rats fed a high-fat diet. Int J Mol Med. 2013;32:1179-86.

33. Wu MH, Huang CC, Chio CC, Tsai KJ, Chang CP, Lin NK, et al. Inhibition of peripheral TNF-alpha and downregulation of microglial activation by alphalipoic acid and etanercept protect rat brain against ischemic stroke. Mol Neurobiol. 2016:53:4961-71.

34. Gorąca A, Huk-Kolega H, Piechota A, Kleniewska P, Ciejka E, Skibska B. Lipoic acid - biological activity and therapeutic potential. Pharmacol Rep. 2011;63: 849-58.

35. Moini H, Packer L, Saris NE. Antioxidant and prooxidant activities of alphalipoic acid and dihydrolipoic acid. Toxicol Appl Pharmacol. 2002;182:84-90.

36. Ho YS, Lai CS, Liu HI, Ho SY, Tai C, Pan MH, et al. Dihydrolipoic acid inhibits skin tumor promotion through anti-inflammation and anti-oxidation. Biochem Pharmacol. 2007:73:1786-95.

37. Calkins MJ, Johnson DA, Townsend JA, Vargas MR, Dowell JA, Williamson TP, et al. The Nrf2/ARE pathway as a potential therapeutic target in neurodegenerative disease. Antioxid Redox Signal. 2009;11:497-508.

38. Patil J, Matte A, Mallard C, Sandberg M. Spirulina diet to lactating mothers protects the antioxidant system and reduces inflammation in post-natal brain after systemic inflammation. Nutr Neurosci. 2018;21(1):59-69.

39. Kyung S, Lim JW, Kim H. alpha-Lipoic acid inhibits IL-8 expression by activating Nrf2 signaling in helicobacter pylori-infected gastric epithelial cells. Nutrients. 2019, 11(10).

40. Lefaki M, Papaevgeniou N, Tur JA, Vorgias CE, Sykiotis GP, Chondrogianni N. The dietary triterpenoid 18alpha-glycyrrhetinic acid protects from MMCinduced genotoxicity through the ERK/Nrf2 pathway. Redox Biol. 2019;28: 101317.

41. Jiang J, Wang Z, Liang $X$, Nie $Y$, Chang $X$, Xue H, et al. Intranasal MMII-0100 attenuates AB1-42- and LPS-induced neuroinflammation and memory impairments via the MK2 signaling pathway. Front Immunol. 2019;10:2707.

42. Zhao C, Hou W, Lei H, Huang L, Wang S, Cui D, et al. Potassium 2-(Ihydroxypentyl)-benzoate attenuates neuroinflammatory responses and upregulates heme oxygenase-1 in systemic lipopolysaccharide-induced inflammation in mice. Acta Pharm Sin B. 2017:7(4):470-8.

43. Yang Z, Jiang $Q$, Chen $S X$, Hu CL, Shen HF, Huang PZ, et al. Differential changes in neuregulin-1 signaling in major brain regions in a lipopolysaccharide-induced neuroinflammation mouse model. Mol Med Rep. 2016;14(1):790-6.

44. Franklin TC, Xu C, Duman RS. Depression and sterile inflammation: essential role of danger associated molecular patterns. Brain Behav Immun. 2018;72 $2-13$.
45. Lugrin J, Martinon F. The AIM2 inflammasome: sensor of pathogens and cellular perturbations. Immunol Rev. 2018;281:99-114.

46. Duncan JA, Canna SW. The NLRC4 inflammasome. Immunol Rev. 2018;281: $115-23$.

47. Sano S, Oshima K, Wang Y, MacLauchlan S, Katanasaka Y, Sano M, et al. Tet2-mediated clonal hematopoiesis accelerates heart failure through a mechanism involving the IL-1beta/NLRP3 inflammasome. J Am Coll Cardiol. 2018;71:875-86.

48. Birnbaum Y, Bajaj M, Yang HC, Ye Y. Combined SGLT2 and DPP4 inhibition reduces the activation of the NIrp3/ASC inflammasome and attenuates the development of diabetic nephropathy in mice with type 2 diabetes. Cardiovasc Drugs Ther. 2018;32:135-45.

49. Scambler T, Jarosz-Griffiths HH, Lara-Reyna S, Pathak S, Wong C, Holbrook J, et al. ENaC-mediated sodium influx exacerbates NLRP3-dependent inflammation in cystic fibrosis. Elife. 2019:8.

50. Gruber JV, Holtz R. In vitro expression of NLRP inflammasome-induced active Caspase-1 expression in Normal human epidermal keratinocytes (NHEK) by various exogenous threats and subsequent inhibition by naturally derived ingredient blends. J Inflamm Res. 2019;12:219-30.

51. Talty A, Deegan S, Ljujic M, Mnich K, Naicker SD, Quandt D, et al. Inhibition of IRE1alpha RNase activity reduces NLRP3 inflammasome assembly and processing of pro-IL1 beta. Cell Death Dis. 2019;10:622.

52. Wang HM, Zhang T, Huang JK, Xiang JY, Chen JJ, Fu JL, et al. Edaravone attenuates the proinflammatory response in amyloid-beta-treated microglia by inhibiting NLRP3 inflammasome-mediated IL-1 beta secretion. Cell Physio Biochem. 2017:43:1113-25.

53. Heid ME, Keyel PA, Kamga C, Shiva S, Watkins SC, Salter RD. Mitochondrial reactive oxygen species induces NLRP3-dependent lysosomal damage and inflammasome activation. J Immunol. 2013;191:5230-8.

54. Raz Y, Neta R, Ronen R. Depression as a microglial disease. Trends Neurosci. 2015;38(10):637-58.

55. Wang X, Zhu L, Hu J, Guo R, Ye S, Liu F, et al. FGF21 attenuated LPSinduced depressive-like behavior via inhibiting the inflammatory pathway. Front Pharmacol. 2020;11:154.

56. Brites D, Fernandes A. Neuroinflammation and depression: microglia activation, extracellular microvesicles and microRNA dysregulation. Front Cell Neurosci. 2015;9:476.

\section{Publisher's Note}

Springer Nature remains neutral with regard to jurisdictional claims in published maps and institutional affiliations.

Ready to submit your research? Choose BMC and benefit from:

- fast, convenient online submission

- thorough peer review by experienced researchers in your field

- rapid publication on acceptance

- support for research data, including large and complex data types

- gold Open Access which fosters wider collaboration and increased citations

- maximum visibility for your research: over $100 \mathrm{M}$ website views per year

At BMC, research is always in progress.

Learn more biomedcentral.com/submissions 